\title{
Mort programmée et terrain cellulaire ${ }^{(1)}$
}

\begin{abstract}
La mort cellulaire semble être l'aboutissement d'un programme partiellement conservé quelles que soient les circonstances inductrices. L'exécution de ce programme de mort, généralement désigné par le terme d'apoptose, dépend de l'état de différenciation des cellules, de la nature de signaux extracellulaires et de la présence de modulateurs intracellulaires, parmi lesquels des oncogènes.
\end{abstract}

\section{Pierre Golstein}

\section{ADRESSE}

P. Golstein : directeur de recherche à l'Inserm. Centre d'immunologie Inserm-Cnrs de Marseille-Luminy, Case 906, 13288 Marseille Cedex 09, France.

(1) Cet article a fait l'objet d'une présentation orale à la 4 e Journée Nationale d'Actualités en Immunologie, organisée par les Laboratoires Cassenne, à Paris, le 15 juin 1991. a mort cellulaire programmée ou apoptose [1] constitue un domaine de recherche en pleine expansion. Le présent point sur ce domaine prend avantage de l'existence de revues [2-7] pour citer surtout des travaux récents, en insistant sur trois notions : l'existence vraisemblable d'un programme commun de mort cellulaire, quel que soit l'agent déclenchant la mort, rendant compte de l'aspect stéréotypé de celle-ci ; mais aussi la multiplicité d'accès et de trajets initiaux intracellulaires vers ce programme, selon les agents ; enfin, et peut-être surtout, l'influence considérable de l'état de différenciation cellulaire (le " terrain " cellulaire) sur l'interprétation d'un signal potentiel de mort cellulaire. Le déclenchement du programme de mort dans la cellule est ainsi conditionné par, à la fois, la survenue d'un signal extracellulaire adéquat et un état approprié du terrain cellulaire.

\section{Mort cellulaire programmée quant à sa survenue}

Le développement normal d'un organe ou d'un système s'effectue non par modelage, mais par sculpture : dans un premier temps, des cellules sont produites en grand excès ; dans un deuxième temps, la plupart de ces cellules meurent, en fonction des critères particuliers requis pour l'élaboration définitive de l'organe ou du système en question. Cette mort cellulaire est clairement programmée quant à sa survenue dans l'organisme, se produisant à un moment donné, pour une cellule donnée, à un endroit donné, suivant des modalités préétablies, de façon raisonnablement prévisible. Le nom d'apoptose évoque précisément la survenue programmée de cette mort, par allusion aux événements programmés responsables de la chute des feuilles à l'automne [1].

La mort cellulaire programmée est un phénomène très général, retrouvé, par exemple, dans des cellules nerveuses et dans divers tissus d'invertébrés. Cependant, le système immunitaire abonde en morts cellulaires induites par différents agents, et c'est ce système qui nous fournira la plupart des modèles ci-dessous. Ce faisant, nous laisserons de côté un autre type de mort cellulaire, appelé nécrose, survenant par exemple lorsque des molécules formant canal, tels certains composants terminaux du complément, attaquent la membrane cellulaire ; et nous considérerons non seulement la mort cellulaire liée au développement, mais aussi d'autres types de mort morphologiquement semblables, survenant en particulier dans certaines situations expérimentales in vitro. 


\section{RÉFÉRENCES}

1. Kerr JFR, Wyllie AH, Currie AR Apoptosis : a basic biological phenomenon with wideranging implications in tissue kinetics. Br J Cancer $1972 ; 26: 239-57$

2. Duvall E, Wyllie AH. Death and the cell. Immunol Today 1986; 7 : 115-9.

3. Kerr JFR, Searle J, Harmon BV, Bishop CJ. Apoptosis. In : Potten CS, ed. Perspectives on Mammalian Cell Dealh. OxfordNew York-Tokyo : Oxford University Press, 1987 : 93-128.

4. Wyllie AH. Apoptosis. ISI Atlas of Science. Immunology 1988 ; 1 : 192-6.

5. Golstein P. Morts cellulaires et système immunitaire. médecine/sciences $1989 ; 5$. 546-53

6. Agid Y, Berke G, Courtois Y, Gol stein P, MacLennan ICM, Prochiantz A. Cell death : mechanisms and functions in development, aging and disease. Philippe Laudal Conferences, 1990. Paris : Éditions Inserm, 1991: 103-70.

7. Golstein P, Ojcius DM, Young JDE Cell death mechanisms and the immune system. Immunol Rev 1991; 121 : 29-65.

8. Hewish DR, Burgoyne LA. Chromatin sub-structure. The digestion of chromatin DNA at regularly spaced sites by a nuclear deoxyribonuclease. Biochem Biophys Res Commun 1973 ; 52 :504-10.

9. Arends MJ, Morris RG, Wyllie AH. Apoptosis : the role of the endonuclease. $A m$ $J$ Pathol 1990 ; 136 : 593-608.

10. Duvall E, Wyllie AH, Morris RG. Macrophage recognition of cells undergoing programmed cell death (apoptosis). Immunology 1985 ; 56 : 351-8.

11. Savill J, Dransfield I, Hogg N, Haslett C. Vitronectin receptor-mediated phagocytosis of cells undergoing apoptosis. Nature 1990 ; 343 : 170-3.

12. Von Boehmer $\mathrm{H}$, Teh HS, Kisielow P. The thymus selects the useful, neglects the useless and destroys the harmful. Immunol Today 1989 ; 10 : 57-61.

13. MacDonald HR, Lees RK. Programmed death of autoreactive thymocytes Nalure 1990 ; 343 : 642-4

14. Collins RJ, Verschuer LA, Harmon BV, Prentice RL, Pope JH, Kerr JFR. Spontaneous programmed death (apoptosis) of B-chronic lymphocytic leukaemia cells following their culture in vitro. $B$ J Haematol $1989 ; 71: 343-50$.

15. Liu YJ, Joshua DE, Williams GT, Smith CA, Gordon J, MacLennan ICM Mechanism of antigen-driven selection in germinal centres. Nalure 1989 ; 342 :

\section{Mort cellulaire programmée quant à son déroulement}

La mort cellulaire par apoptose semble programmée, non seulement quant à sa survenue dans l'organisme, mais aussi quant à son déroulement dans la cellule (figure 1). Deux des étapes de ce déroulement sont constamment retrouvées : la fragmentation précoce et régulière de l'ADN, et l'aspect morphologique caractéristique de condensation/fragmentation nucléaire et cytoplasmique.

La fragmentation de l'ADN de cellules mourantes en fragments de 180-200 paires de bases et de leurs multiples est démontrée par un aspect caractéristique " en échelle " de l'ADN en électrophorèse en gel. Ces fragments sont le résultat de la digestion, d'abord partielle, de l'ADN génomique par des endonucléases agissant sur des sites exposés entre les nucléosomes [8]. Une telle fragmentation de l'ADN a pu être mimée dans un système modèle, la digestion in vitro de noyaux de thymocytes de rat par une nucléase en présence d'inhibiteurs de protéases [9].

Morphologiquement, la mort par apoptose est un processus de condensation/fragmentation. Une caractéristique précoce et remarquable en est la condensation du cytoplasme, avec préservation de la morphologie des mitochondries et des ribosomes, et perte des microvillosités de surface. La condensation de la chromatine et sa marginalisation conduisent à la formation de masses de chromatine à bord net alignées le long de la membrane nucléaire. Des invaginations marquées de la membrane nucléaire conduisent à la fragmentation du noyau, tandis que les invaginations de la membrane plasmique conduisent à la fragmentation cytoplasmique. Cela aboutit à la constitution d'amas ("corps apoptotiques") de fragments encore entourés par une membrane et excluant le bleu trypan. Ces fragments cellulaires sont très rapidement éliminés des tissus par phagocytose, ce qui contribue à rendre difficile la détection dans un tissu d'un taux même important d'apoptose; la reconnaissance de cellules mourantes ou mortes par des phagocytes a été attribuée à des molécules de type lectine à la surface des phagocytes [10], et/ou à la vitronectine et à son récepteur [11].

Cet aspect stéréotypé de la mort cellulaire programmée, quel qu'en soit l'agent causal, semble refléter un mécanisme moléculaire terminal unique. La nature de l'agent causal influence des caractéristiques surajoutées, en particulier la rapidité du phénomène. Par ailleurs, c'est l'aspect stéréotypé de ces morts cellulaires, correspondant à un déroulement programmé dans la cellule, qui très souvent maintenant, par distorsion étymologique, définit l'apoptose. Une mort " par apoptose " désignera ainsi une mort cellulaire ayant les caractéristiques morphologiques indiquées ci-dessus, même si cette mort survient en dehors de tout contexte de développement.

\section{De multiples agents peuvent déclencher la mort par apoptose}

L'accès au mécanisme moléculaire terminal unique de l'apoptose peut être multiple. On considérera essentiellement les modèles in vitro mettant en évidence cette multiplicité d'agents capables de déclencher la mort cellulaire.

La stimulation $\mathrm{du}$ complexe TcR/CD3, le récepteur pour l'antigène localisé à la surface des lymphocytes $T$, peut entraîner la mort par apoptose de certains de ces lymphocytes, notamment des thymocytes immatures. In vitro, cette stimulation est obtenue lorsque le complexe TcR/CD3 est lié, soit par son ligand "physiologique ", l'antigène, soit par un superantigène, soit par un anticorps anti-TcR ou antiCD3 (figure 1). Ces modèles miment très vraisemblablement l'élimination massive in vivo, dans le thymus, de lymphocytes immatures porteurs de récepteurs anti-soi, élimination contribuant à éviter une réactivité autoimmune ultérieure [12-13]. Il est important de noter d'emblée que la même stimulation, mais s'exerçant sur un lymphocyte $\mathrm{T}$ mûr, conduit en règle générale non pas à la mort, mais bien au contraire à la prolifération et à la différenciation de ce lymphocyte. 


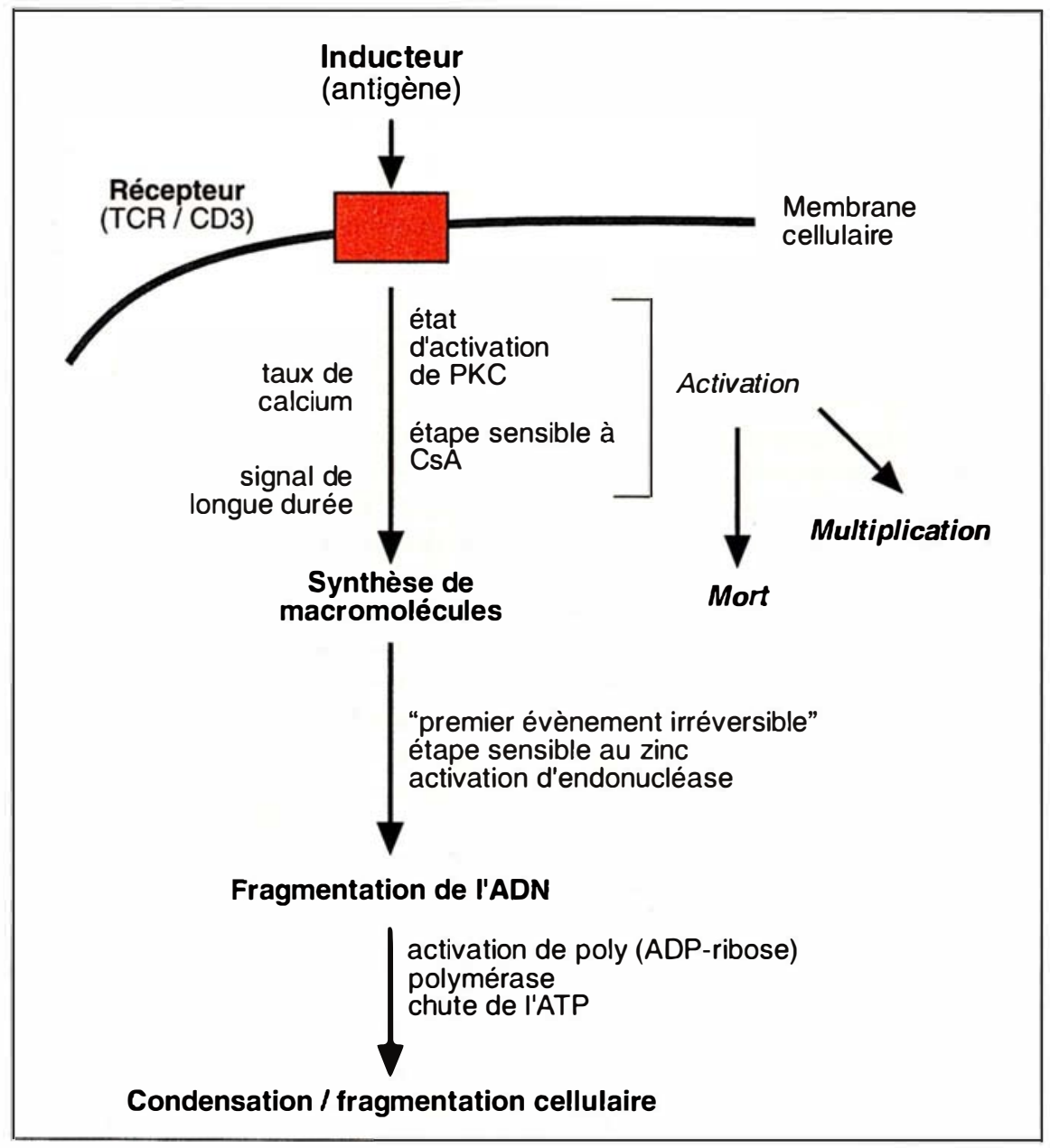

Figure 1. L'apoptose est programmée dans son déroulement intracellulaire. L'exemple montré ici (adapté de [7]) représente un thymocyte reconnaissant un antigène du soi ; l'activation qui en résulte se traduit chez ce lymphocyte immature par un aiguillage vers la mort. Pour plus de détails sur les différentes étapes du programme intracellulaire de mort, voir le texte et [7]. $P K C=$ protéine kinase $C ; C s A$ : ciclosporine $A$.

La mort par apoptose de lymphocytes B [14-17] peut se produire dans des circonstances très voisines de celles qui sont observées dans la lignée T. Suivant le stade de différenciation des cellules B, l'occupation des immunoglobulines de surface servant de récepteurs pour l'antigène peut déclencher [16-18] ou prévenir [15] la mort par apoptose de ces lymphocytes B. Dans ce dernier cas, des lymphocytes B de centres germinatifs amygdaliens meurent "spontanément " par apoptose à leur mise en culture in vitro ; il s'agit majoritairement de cellules positives pour le marqueur CD77, un glycolipide neutre $[19,20]$; la mort de ces $m / s n^{\circ} 7$, vol. 7 , septembre 91 être induite par le tumor necrosis factor alpha $(\mathrm{TNF} \alpha)$, une protéine de $17 \mathrm{kDa}$, cytotoxique pour nombre de lignées cellulaires en culture mais aussi capable non seulement de ne pas tuer, mais même de stimuler certaines autres cellules. Au TNF $\alpha$ correspondent des récepteurs spécifiques à la surface cellulaire. Un point essentiel est que des anticorps, dirigés contre ces récepteurs [21] ou contre la molécule Fas probablement associée à ces récepteurs [22, 23], déclenchent chez les cellules sensibles une mort cellulaire semblable à celle qui est déclenchée par le $\mathrm{TNF} \alpha$ luimême. La molécule importante pour la transmission d'un signal de mort dans la cellule est donc ce récepteur, qu'il soit stimulé par son ligand naturel, le $\mathrm{TNF} \alpha$, ou par des anticorps. Les récepteurs pour le $\mathrm{TNF} \alpha$ sont présents à la surface de cellules sensibles, mais également à la surface de cellules résistantes à la lyse induite par le $\mathrm{TNF} \alpha$, ce qui suggère que, là aussi, ce sont les conditions intracellulaires qui vont faire qu'un certain signal entraînera ou non la mort. Des structures de surface cellulaire autres que les récepteurs spécifiques de l'antigène ou du $\mathrm{TNF} \alpha$ peuvent transduire un signal de mort vers l'intérieur de la cellule qui les porte. C'est ainsi, par exemple, que la mort cellulaire peut être déclenchée par des anticorps dirigés contre la molécule de surface APO-1 [24, 25], dont on ne connaît pas encore le ligand naturel. Des récepteurs de surface pour des interleukines ou des hormones peuvent également moduler la mort cellulaire. Par exemple, la mort par apoptose peut résulter d'un déficit en interleukines et être prévenue par leur addition [26-30] ; cela peut être rapproché d'autres observations, dans le système immunitaire et en dehors de ce système, où des facteurs de croissance et des hormones préviennent la mort cellulaire [31-34], ou dans lesquelles des facteurs solubles encore non identifiés y contribuent $[35,36]$.

Des altérations intracellulaires peuvent aussi conduire à la mort cellulaire. La destruction de thymocytes par des corticoïdes constitue ainsi un modèle classique de mort cellulaire par apoptose. Moins classiques, mais au moins aussi révélatrices quant au 
contrôle de la mort cellulaire, sont les morts cellulaires induites par des drogues provoquant une altération ou un découplage métabolique [37-41]. Par exemple, pour certaines cellules, le simple blocage de la synthèse de

16. Benhamou LE, Cazenave PA, Sarthou P. Anti-immunoglobulins induce death by apoptosis in WEHI-231 B lymphoma cells. Eur J Immunol 1990 ; 20 : 1405-7.

17. Hasbold J, Klaus GGB. Antiimmunoglobulin antibodies induce apoptosis in immature B-cell lymphomas. Eur $J$ Immunol 1990 ; 20 : 1685-90.

18. McConkey DJ, Aguilar-Santelises $M$, Hartzell $\mathrm{P}$, et al. Induction of DNA fragmentation in chronic B-lymphocytic leukemia cells. J Immunol 1991; 146 : 1072-6.

19. Mangeney M, Richard Y, Coulaud D, Tursz T, Wiels J. CD77 : an antigen of germinal center B-cells entering apoptosis. Eur J Immunol 1991; 21 : 1131-40.

20. Liu YJ, Cairns JA, Holder MJ, et al. Recombinant $25-\mathrm{kDa}$ CD23 and interleukin 1 alpha promote the survival of germinal center B-cells : evidence for bifurcation in the development of centrocytes rescued from apoptosis. Eur J Immunol 1991; 21 : 1107-14.

21. Espevik T, Brockhaus M, Loetscher $\mathrm{H}$, Nonstad U, Shalaby R. Characterization of binding and biological effects of monoclonal antibodies against a human tumor necrosis factor receptor. $J$ Exp Med $1990 ; 171$ 415-26.

22. Yonehara S, Ishii A, Yonehara M. cellkilling monoclonal antibody (anti-Fas) to a cell surface antigen co-downregulated with the receptor of tumor necrosis factor. $J$ Exp Med $1989 ; 169$ : 1747-56.

23. Hashimoto S, Ishii A, Yonehara S. The E1b oncogene of adenovirus confers cellular resistance to cytotoxicity of tumor necrosis factor and monoclonal anti-Fas antibody. Int Immunol $1991 ; 3$ : 343-51.

24. Trauth BC, Klas C, Peters AMJ, et al. Monoclonal antibody-mediated tumor regression by induction of apoptosis. Science $1989 ; 245: 301-5$

25. Debatin KM, Goldmann CK, Bamford R, Waldmann TA, Krammer PH. Monoclonal-antibody-mediated apoptosis in adult T-cell leukaemia. Lancet 1990 ; 335 : 497-500.

26. Cohen JJ, Duke RC, Chervenak R, Sellins KS, Olson LK. DNA fragmentation in targets of CTL : an example of programmed cell death in the immune system. $A d v$ Exp Med Biol 1985 ; 184：493-508.

27. Duke RC, Cohen JJ. IL-2 addiction: withdrawal of growth factor activates a suicide program in dependent T-cells. Lympho-
28. Fernández-Ruiz E, Rebollo A, Nieto MA, et al. IL-2 protects T-cell hybrids from the cytolytic effect of glucocorticoids : Synergistic effect of IL-2 and dexamethasone in the induction of high-affinity IL-2 receptors. J Immunol 1989 ; 143 : 4146-51.

29. Nieto MA, López-Rivas A. IL-2 protects $\mathrm{T}$ lymphocytes from glucocorticoidinduced DNA fragmentation and cell death J Immunol 1989 ; 143 : 4166-70.

30. McConkey DJ, Hartzell P, Chow SC, Orrenius S, Jondal M. Interleukin 1 inhibits $\mathrm{T}$-cell receptor-mediated apoptosis in immature thymocytes. J Biol Chem 1990 ; $265: 3009-11$

31. Martin DP, Schmidt RE, DiStefano PS, Lowry OH, Carter JG, Johnson EM Jr. Inhibitors of protein synthesis and RNA synthesis prevent neuronal death caused by nerve growth factor deprivation. J Cell Biol 1988 ; 106 : 829-44.

32. Terada N, Yamamoto $\mathrm{R}$, Takada $\mathrm{T}$, et al. Inhibitory effect of progesterone on cell death of mouse uterine epithelium. J Steroid Biochem 1989 ; 33 : 1091-6.

33. Williams GT, Smith CA, Spooncer E, Dexter TM, Taylor DR. Haemopoietic colony stimulating factors promote cell survival by suppressing apoptosis. Nature 1990 ; $343: 76-9$

34. Koury MJ, Bondurant MC. Erythropoietin retards DNA breakdown and prevents programmed cell death in erythroid progenitor cells. Science $1990 ; 248$ : 378-81.

35. Kosaka H, Ogata M, Hikita I, et al. Model for clonal elimination in the thymus. Proc Nall Acad Sci USA 1989 ; 86 : 3773-7.

36. Pierce GB, Lewellyn AL, Parchment RE. Mechanism of programmed cell death in the blastocyst. Proc Natl Acad Sci USA 1989 ; 86 : 3654-8.

37. Searle J, Lawson TA, Abbott PJ, Harmon B, Kerr JFR. An electron-microscope study of the mode of cell death induced by cancer-chemotherapeutic agents in populations of proliferating normal and neoplastic cells. J Pathol 1975; 116 : 129-38.

38. Kolber MA, Broschat $\mathrm{KO}$, LandaGonzalez B. Cytochalasin B induces cellular DNA fragmentation. FASEB J $1990 ; 4$ : 3021-7.

39. Kung AL, Zetterberg A, Sherwood SW, Schimke RT. Cytotoxic effects of cell cycle phase specific agents : result of cell cycle perturbation. Cancer Res 1990 ; 50 : 7307-17. macromolécules suffit à déclencher la mort par apoptose [37, 42, 43], suggérant l'inhibition par des molécules à très courte demi-vie d'un programme interne de mort cellulaire. D'autres molécules à très courte demi-vie pourraient, au contraire, être requises pour le déclenchement de la mort cellulaire [44]. Il est possible qu'existe, dans certaines cellules, un équilibre entre molécules à courte demi-vie déclenchant ou prévenant la mort cellulaire. Cet équilibre peut être fragile, expliquant pourquoi des agressions très diverses et parfois assez modérées peuvent conduire au déclenchement de la mort cellulaire.

Une mort cellulaire ayant les caractéristiques de l'apoptose peut aussi être causée par des cellules $\mathrm{T}$ cytotoxiques (CTL). La nature des CTL et ce que l'on connaît de leurs mécanismes de lyse ont récemment fait l'objet de revues [45-47] ; plusieurs molécules produites par les CTL dont la perforine, les TNF et, plus récemment, l'ATP [48-50] — ont été proposées comme pouvant jouer un rôle dans la lyse de la cellule cible. Dans une cellule cible tuée par une cellule tueuse, après une augmentation brusque et marquée du taux de calcium cytoplasmique, apparaissent une fragmentation de l'ADN en segments de 180-200 pb et une condensation/fragmentation cytoplasmique et nucléaire. Ces traits sont similaires à ceux qui sont observés dans la mort cellulaire au cours du développement ou induite par le $\mathrm{TNF} \alpha$, sauf en ce qui concerne leur cinétique d'apparition - remarquablement rapide, des minutes au lieu d'heures.

\section{Plusieurs types de mort par apoptose}

Suivant l'agent causal considéré, la mort cellulaire peut présenter, sur un fond commun d'apoptose, des traits particuliers. Ceux-ci peuvent refléter des différences dans les trajets allant du récepteur de l'agent causal vers le mécanisme commun terminal de 
l'apoptose, comme analysé en détail récemment [7]. Des sous-catégories d'apoptose peuvent être individualisées (Tableau I), tels le type I pour l'apoptose associée au développement et pour l'apoptose " transduite " par le TcR/CD3, ou causée par des corticoïdes ou par irradiation; le type II pour l'apoptose " transduite" par le récepteur pour le $\mathrm{TNF} \alpha$; et le type III pour la mort par apoptose causée par des cellules cytotoxiques $\mathrm{T}$.

Ces types d'apoptose different, en particulier, par le temps nécessaire entre l'application de l'agent déclenchant la mort et cette mort : beaucoup d'agents entraînent la mort cellulaire en quelques heures (les expériences sur la mort cellulaire induite par corticoïdes, irradiation ou certains anticorps - apoptoses de type I et II - impliquent typiquement des incubations sur la nuit), d'autres n'ont besoin pour entraîner la mort cellulaire que de quelques minutes (apoptose de type III).

Ces types d'apoptose diffèrent également par certaines étapes métaboliques nécessaires entre l'application de l'agent et la mort. Ainsi, une synthèse de macromolécules est-elle nécessaire dans l'apoptose de type I, qui peut être inhibée par des inhibiteurs de synthèse d'ARN comme l'actinomycine $\mathrm{D}$, ou de synthèse de protéines comme la cycloheximide [31, 51-54] ; dans ce type d'apoptose, la cellule qui meurt doit donc activement synthétiser certaines macromolécules pour mourir.

En apparent contraste, dans l'apoptose de type II, non seulement la synthèse de macromolécules n'est pas nécessaire, mais la sensibilité au $\mathrm{TNF} \alpha$ est souvent considérablement augmentée par prétraitement des cellules cibles avec des inhibiteurs de synthèse de macromolécules comme l'actinomycine D ou la cycloheximide $[55,56]$, suggérant que les cellules peuvent prévenir les lésions induites par le TNF $\alpha$ en synthétisant des protéines protectrices. Celles-ci peuvent préexister dans certaines cellules, rendant compte des différences de sensibilité entre cellules et de la dominance du phénotype "résistant" dans des hybrides somatiques [57]. Une de ces protéines protectrices néo-synthétisées semble être la $\mathrm{m} / \mathrm{s} n^{\circ} 7$, vol. 7 , septembre 91

Tableau 1

DIFFÉRENTS TYPES DE MORT PAR APOPTOSE

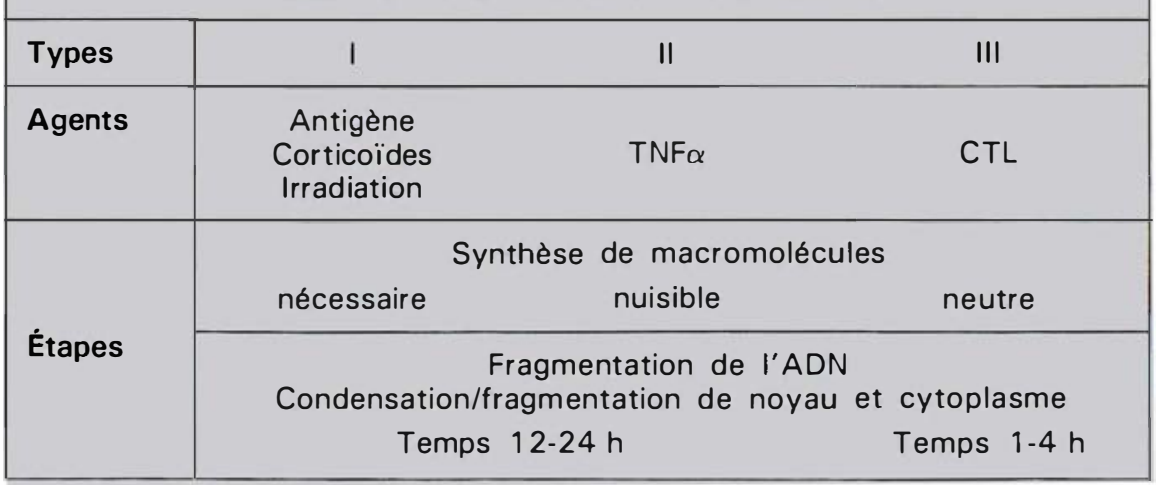

Adapté de [7].

$C T L=$ cytotoxic $\mathrm{T}$ lymphocytes.

$T N F \alpha=$ tumor necrosis factor alpha.

manganèse-superoxyde dismutase (une protéine mitochondriale impliquée dans l'élimination des radicaux $\mathrm{O}^{-}$), dont la surexpression protège, et dont un ARNm antisens sensibilise, les cellules au TNF $\alpha$ [58].

Les inhibiteurs de synthèse de macromolécules n'inhibent pas la lyse de cellules cibles par des CTL (apoptose de type III) quand ils sont ajoutés au début du test de cytotoxicité $[59,60]$. Ainsi, aucune protéine, néosynthétisée dans la cible après addition de CTL, ne semble nécessaire à la lyse de la cible. Cependant, la synthèse continue par la cellule cible de molécules préexistantes et à courte durée de vie peut être nécessaire à la lyse de certaines cellules cibles; en effet, leur préincubation pendant plusieurs heures avec des inhibiteurs de synthèse de protéine ou de l'ARN conduit à une diminution marquée de leur capacité à être ensuite lysées [60,61].

D'autres différences métaboliques peuvent exister entre les divers types d'apoptose. Ainsi, il n'y a pas de nécessité de calcium extracellulaire ni intracellulaire dans l'apoptose de type II [62], ni dans certains cas d'apoptose de type III. Par ailleurs, l'apoptose de type II, et plus précisément la capacité d'une cellule à résister au TNF $\alpha$, semble dépendre de sa capacité à compenser une attaque oxydative [63], en accord avec les résultats sur la manganèse- superoxyde dismutase mentionnés plus haut.

Les voies intracellulaires conduisant à l'apoptose peuvent être différentes même à l'intérieur de certains des types individualisés dans le Tableau I. Ainsi, comme indiqué plus haut, l'apoptose de thymocytes peut être déclenchée soit par un anticorps antiTcR/CD3, soit par un corticoïde; de façon frappante, cependant, si les deux agents sont appliqués simultanément, les thymocytes ne meurent pas, suggérant que les voies de contrôle intracellulaires correspondantes non seulement sont différentes, mais encore interferrent négativement [64, 65].

Toutes ces particularités de cinétique et d'exigences métaboliques dans la cellule qui va mourir peuvent être dues à des différences, non pas dans le mécanisme de mort par apoptose proprement dit, mais dans un compartiment contrôle, avant le déclenchement du mécanisme d'apoptose et le conditionnant (figure 2). Il est très remarquable que l'apoptose de type III, c'est-à-dire la mort causée par une cellule cytotoxique, se déroule très rapidement et semble comporter un minimum d'exigences métaboliques. Cela suggère que le compartiment de contrôle y est d'importance réduite. La mort par cellules cytotoxiques pourraient ainsi être, pour l'étude du mécanisme de l'apoptose, un modèle privilégié, car 


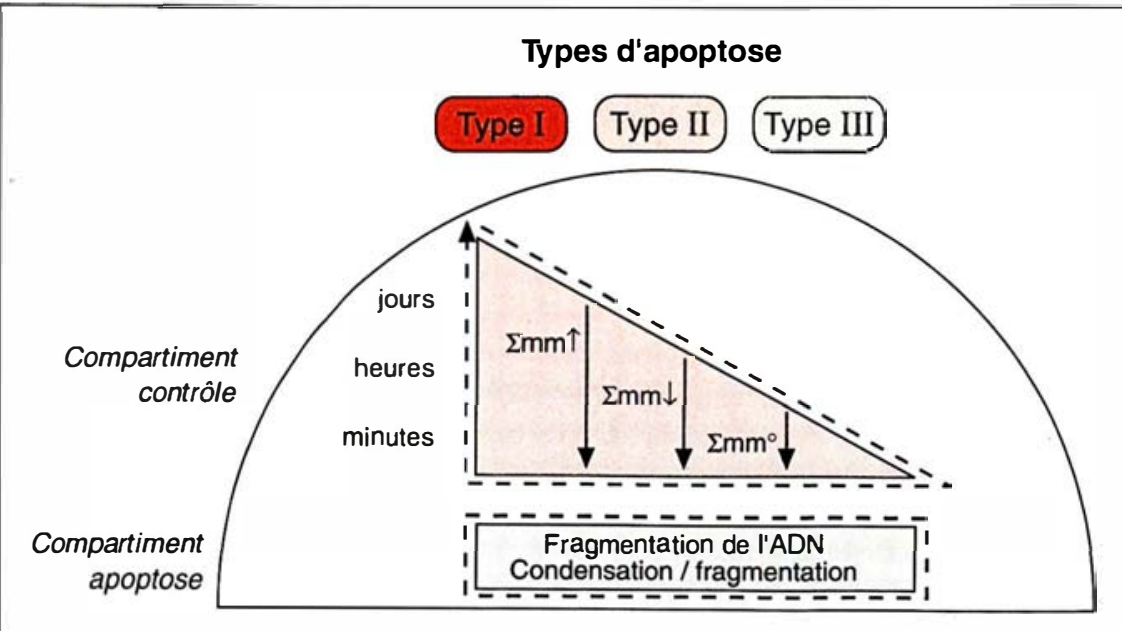

40. Barry MA, Behnke CA, Eastman A Activation of programmed cell death (apoptosis) by cisplatin, other anticancer drugs, toxins and hyperthermia. Biochem Pharmacol $1990 ; 40: 2353-62$.

41. Bansal N, Houle A, Melnykovych G. Apoptosis : mode of cell death induced in T-cell leukemia lines by dexamethasone and other agents. FASEB J $1991 ; 5$ : 211-6.

42. Martin SJ, Lennon SV, Bonham AM, Cotter TG. Induction of apoptosis (programmed cell death) in human leukemic HL- 60 cells by inhibition of RNA or protein synthesis. J Immunol 1990; 145 : 1859-67.

43. Cohen JJ. Programmed cell death in the immune system. Adv Immunol 1991; (sous presse).

44. McConkey DJ, Hartzell P, Orrenius S, Rapid turnover of endogenous endonuclease activity in thymocytes : effects of inhibitors of macromolecular synthesis. Arch Biochem Biophys 1990 ; 278 : 284-7.

45. Berke G. Functions and mechanisms of lysis induced by cytotoxic $\mathrm{T}$ lymphocytes and natural killer cells. In : Paul WE, ed. Fundamental Immunology. New York: Raven Press, 1989 : 735-64.

46. Young JDE. Killing of target cells by lymphocytes : a mechanistic view. Physiol Rev $1989 ; 69: 250-314$

47. Tschopp J, Nabholz M. Perforinmediated target cell lysis by cytolytic $\mathrm{T}$ lymphocytes. Ann Rev Immunol 1990; 8 : 279-302.

48. Filippini A, Taffs RE, Sitkovsky MV. Extracellular ATP in T-lymphocyte activation : possible role in effector functions. Proc Nall Acad Sci USA 1990; 87 : 8267-71.

49. Di Virgilio F, Pizzo P, Zanovello P, Bronte V, Collavo D. Extracellular ATP as a possible mediator of cell-mediated cytotoxicity. Immunol Today $1990 ; 11: 274-7$.

50. Zheng LM, Zychlinsky A, Liu CC, Ojcius DM, Young JDE. Extracellular ATP as a trigger for apoptosis or programmed cell death. J Cell Biol 1991; 112 : 279-88.

51. Cohen JJ, Duke RC. Glucocorticoid activation of a calcium-dependent endonuclease in thymocyte nuclei leads to cell death. J Immunol 1984; 132 : 38-42.

52. Wyllie AH, Morris RG, Smith AL, Dunlop D. Chromatin cleavage in apoptosis : association with condensed chromatin morphology and dependence on macromolecular synthesis. J Pathol 1984; 142: 67-77. parasité par un minimum d'événements contrôle.

\section{Le terrain cellulaire conditionne l'apoptose}

La transduction d'un même message potentiel de mort cellulaire pourra conduire à un résultat très différent selon le terrain cellulaire et en particulier selon le stade de différenciation de la cellule. Ainsi, l'occupation (par l'antigène ou par un anticorps spécifique) du complexe TcR/CD3 transduit vers l'intérieur de la cellule un certain signal. Ce signal conduit à un état d'activation aboutissant classiquement soit à la mort (pour un thymocyte), soit à la multiplication cellulaire (pour un lymphocyte $\mathrm{T}$ mûr). Une situation analogue existe pour les lymphocytes $B$ recevant un signal par l'intermédiaire des Ig de surface. Également, le $\mathrm{TNF} \alpha$ peut stimuler certaines cellules et en tuer d'autres. Il ne s'agit donc pas là (et il n'existe peut-être pas) de signaux extracellulaires de mort auxquels toutes les cellules répondraient de façon univoque, mais de signaux conditionnels, dont la signification est modulée par l'état des cellules réceptrices.

\section{Terrain cellulaire, apoptose et protéines du HIV}

Fait très important, il a été récemment démontré que, non seulement des thymocytes, mais aussi des lymphocytes $\mathrm{T}$ mûrs peuvent être induits par stimulation du TcR/CD3 à mourir par apoptose, s'ils ont été, de façon adéquate, préstimulés ou costimulés [66-68]. En particulier, des lymphocytes T CD4 + spléniques de souris, s'ils sont d'abord incubés avec des anticorps anti-CD4 dans certaines conditions, peuvent être induits par des anticorps anti-TcR/CD3 à mourir par apoptose [69]. Ce dernier résultat a de fascinantes implications. Sur un plan fondamental, il indique que le prétraitement par des anticorps anti-CD4 modifie l'état des lymphocytes $\mathrm{T}$ de telle sorte que le message transmis par le TcR/CD3 sera interprété dans le sens de l'apoptose. Sur un plan plus appliqué et récemment discuté en détail [70], ce résultat peut fournir une explication au paradoxe apparent de la mort, chez des malades du SIDA, de la majorité des lymphocytes $T$ CD4 + , alors qu'une petite minorité seulement de ces cellules est infectée 
par le HIV (m/s $n^{\circ} 6$, vol. 7, p. 604). Dans un premier temps (figure 3 ), les lymphocytes T CD4 + seraient préstimulés par interaction de leurs molécules de surface CD4 et des protéines gp120 du HIV (circulantes ou présentées sur des cellules infectées par le HIV). Les lymphocytes T CD4 +, ainsi préstimulés par l'intermédiaire de leur CD4, seraient sensibilisés à une éventuelle stimulation ultérieure par l'intermédiaire de leur TcR. Cette stimulation se produira à l'occasion, par exemple, d'une infection, lorsque le $\mathrm{TcR}$ sera engagé par l'antigène correspondant ou par un éventuel superantigène bactérien : cet engagement conduira alors à une mort par apoptose, alors que le même engagement chez un lymphocyte mûr non préstimulé aurait normalement conduit à une réponse de type multiplication.

\section{Terrain cellulaire, apoptose et oncogènes}

Également intéressante et relativement récente est la notion d'interférence oncogène/mort cellulaire. Ainsi, le candidat oncogène $b c l-2$, découvert par sa localisation chromosomique flanquant une translocation dans un lymphome B [71] et codant pour une protéine mitochondriale [72], prévient $[72,73]$ la mort par apoptose survenant dans certaines lignées cellulaires par privation d'IL-3 $\left(\mathrm{m} / \mathrm{s} n^{\circ} 1\right.$, vol. 7, p. 88). Le mécanisme de la prévention de la mort cellulaire par le produit de bcl-2 n'est pas encore connu. L'apoptose de cellules B humaines peut également être prévenue par l'activation de certains gènes latents du virus d'Epstein-Barr [74].

Un phénomène apparemment similaire est la modulation de la sensibilité au TNF $\alpha$ par expression d'oncogènes. Cette sensibilité est diminuée par expression de l'oncogène HER2/neu dans des cellules NIH 3T3 [75] et dans diverses cellules tumorales humaines [76, 77]. Par ailleurs, les produits de deux gènes distincts d'adénovirus respectivement augmentent [78] ou diminuent [79] la sensibilité cellulaire au TNF $\alpha$. La protéine de l'oncogène E1b de l'adénovirus type 2 confère à des cellules, normalement sensibles au TNF $\alpha$ et à des anticorps anti-Fas, une résis$\mathrm{m} / \mathrm{s} n^{\circ} 7$, vol. 7, septembre 91

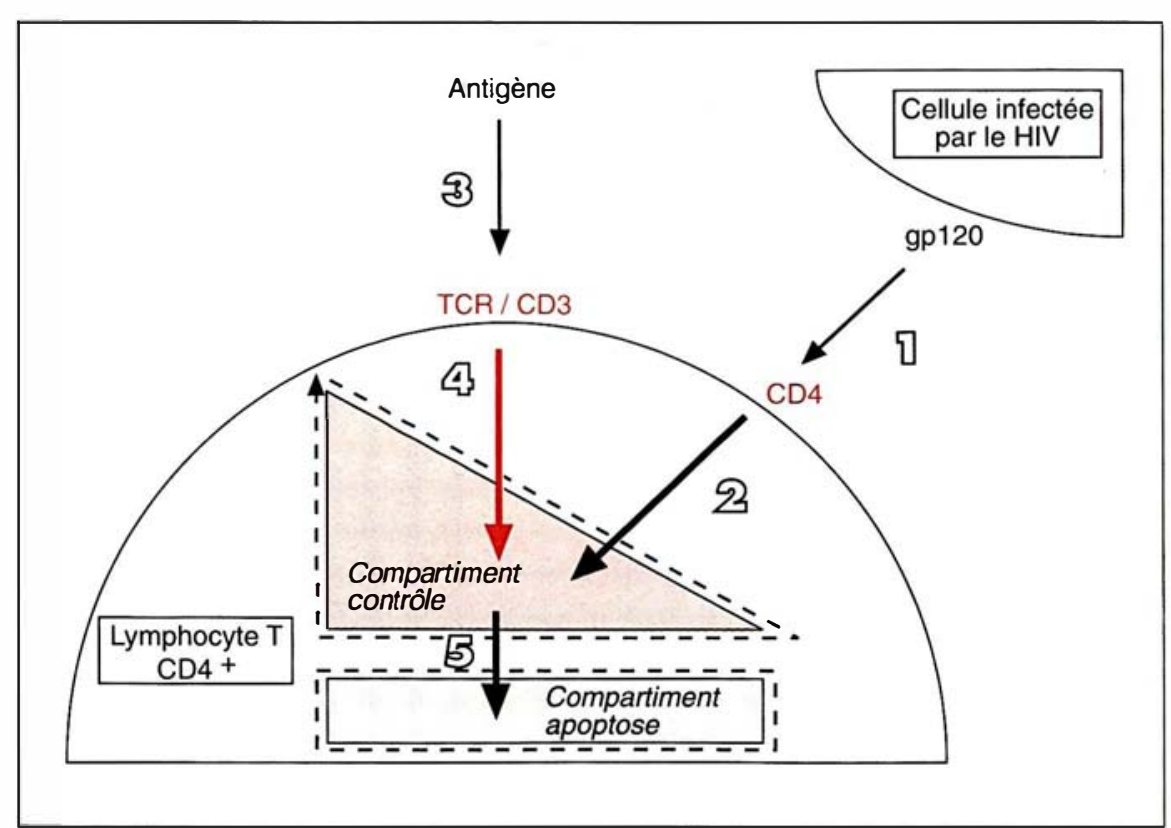

Figure 3. Un mode possible de mort cellulaire chez un patient infecté par le HIV. Ce schéma est fondé sur les résultats de [69], également discutés dans [70]. La protéine gp120 à la surface de cellules infectées par le HIV, ou circulante, se lie (1) à une molécule CD4 à la surface d'un lymphocyte $T$ $\mathrm{CD}^{+}{ }^{+}$. Cela entraîne (2) une altération du terrain cellulaire, modifiant en particulier le compartiment contrôle. Le signal transmis (4) par le TCR/CD3 stimulé par un antigène (3) sera alors interprété comme un signal de mort (5).

tance marquée à ces deux agents [23] ; or, l'expression de cet oncogène conditionne la régulation de divers événements intracellulaires, comme le taux d'expression de la manganèse superoxyde dismutase [23], dont nous avons vu plus haut l'impact sur la résistance cellulaire à des radicaux $\mathrm{O}^{-}$et, vraisemblablement, corrélativement, au TNF $\alpha$. Le transforming growth factor $\beta 1$ induit également l'apoptose de certaines cellules [80], qui peuvent lui devenir résistantes par expression de l'oncogène $\mathrm{E} 1 \mathrm{~A}$ d'adénovirus [81].

Ces résultats ont, là aussi, des implications multiples. En cancérogenèse, un premier oncogène empêchant la mort cellulaire pourrait favoriser la transformation maligne, par exemple en rendant possible l'intervention ultérieure, en série, d'un deuxième oncogène. En dehors de la cancérogenèse, le rôle des produits de ces oncogènes prévenant la mort cellulaire demeure mystérieux. Par ailleurs, l'étude de la façon dont certains de ces oncogènes interfèrent avec les processus d'apoptose peut fournir une précieuse (car rare) prise expérimentale sur les mécanismes moléculaires de ces processus

TIRÉS A PART

P. Golstein 
53. Sellins KS, Cohen JJ. Gene induction by gamma-irradiation leads to DNA fragmentation in lymphocytes. J Immunol 1987; 139 : 3199-3206.

54. Yamada T, Ohyama H. Radiationinduced interphase death of rat thymocytes is internally programmed (apoptosis). Int J Radiat Biol 1988 ; 53 : 65-75.

55. Ruff MR, Gifford GE. Rabbit tumor necrosis factor : mechanism of action. Infect Immun $1981 ; 31: 380-5$.

56. Kirstein M, Baglioni C. Tumor necrosis factor induces synthesis of two proteins in human fibroblasts. $J$ Biol Chem 1986 ; 261: 9565-7.

57. Nophar Y, Holtmann H, Ber R, Wallach D. Dominance of resistance to the cytocidal effect of tumor necrosis factor in heterokaryons formed by fusion of resistant and sensitive cells. J Immunol $1988 ; 140$ : 3456-60.

58. Wong GHW, Elwell JH, Oberley LW, Goeddel DV. Manganous superoxide dismutase is essential for cellular resistance to cytotoxicity of tumor necrosis factor. Cell 1989 ; 58 : 923-31.

59. Duke RC, Chervenak R, Cohen JJ. Endogenous endonuclease-induced DNA fragmentation: an early event in cellmediated cytolysis. Proc Natl Acad Sci USA $1983 ; 80: 6361-5$

60. Landon C, Nowicki M, Sugawara S, Dennert G. Differential effects of protein synthesis inhibition on CTL and targets in cell-mediated cytotoxicity. Cellular Immunol $1990 ; 128$ : 412-26.

61. Zychlinsky A, Zheng LM, Liu CC, Young JDE. Cytotoxic lymphocytes induce both apoptosis and necrosis in target cells. J Immunol 1991; 146 : 393-400.

62. Hasegawa Y, Bonavida B. Calciumindependent pathway of tumor necrosis factor-mediated lysis of target cells. J Immunol $1989 ; 142$ : 2670-6.

63. Zimmerman RJ, Marafino Jr BJ, Chan A, Landre P, Winkelhake JL. The role of oxidant in jury in tumor cell sensitivity to recombinant human tumor necrosis factor in vivo. Implications for mechanisms of action. J Immunol 1989; 142 : 1405-9.

64. Zacharchuk CM, Mercep M, Chakraborti PK, Simons SS Jr, Ashwell JD. Programmed $T$ lymphocyte death. Cell activation- and steroid-induced pathways are mutually antagonistic. J Immunol 1990 ; $145: 4037-45$

65. Iwata M, Hanaoka S, Sato K. Rescue of thymocytes and $\mathrm{T}$-cell hybridomas from glucocorticoid-induced apoptosis by stimulation via the T-cell receptor/CD3 complex: tion of the T-cell repertoire. Eur J Immunol $1991 ; 21: 643-8$.

66. Russell JH, White CL, Loh DY, Meleedy-Rey P. Receptor-stimulated death pathway is opened by antigen in mature $\mathrm{T}$ cells. Proc Nall Acad Sci USA 1991; 88 : 2151-5.

67. Janssen $O$, Wesselborg $S$ HecklÖstreicher B, el al. T-cell receptor/CD3-signaling induces death by apoptosis in human $\mathrm{T}$-cell receptor gamma delta ${ }^{+}$T-cells. J Immunol 1991; 146 : 35-9.

68. Lucas M, Solano F, Sanz A. Induction of programmed cell death (apoptosis) in mature lymphocytes. FEBS Lell 1991; 279 : 19-20.

69. Newell MK, Haughn LJ, Maroun CR, Julius $\mathrm{MH}$. Death of mature T-cells by separate ligation of CD4 and the T-cell receptor for antigen. Nature $1990 ; 347$ : 286-9.

70. Ameisen JC, Capron A. Cell dysfunction and depletion in AIDS : the programmed cell death hypothesis. Immunol Today $1991 ; 12: 102-5$.

71. Tsujimoto $\mathrm{Y}$, Finger LR, Yunis $\mathrm{J}$ Nowell PC, Croce CM. Cloning of the chromosome breakpoint of neoplastic B-cells with the $\mathrm{t}(14 ; 18)$ chromosome translocation. Science 1984; 226 : 1097-9.

72. Hockenbery D, Nunez G, Milliman C, Schreiber RD, Korsmeyer SJ. Bcl-2 is an inner mitochondrial membrane protein that blocks programmed cell death. Nature 1990 ; $348: 334-6$

73. Vaux DL, Cory S, Adams JM. Bcl-2 gene promotes haemopoietic cell survival and cooperates with $c-m y c$ to immortalize pre-B cells. Nature 1988 ; 335 : 440-2.

74. Gregory CD, Dive C, Henderson S, et al. Activation of Epstein-Barr virus latent genes protects human B-cells from death by apoptosis. Nature 1991; 349: 612-4.

75. Hudziak RM, Lewis GD, Shalaby MR, el al. Amplified expression of the HER2/ERBB2 oncogene induces resistance to tumor necrosis factor alpha in NIH 3T3 cells. Proc Nall Acad Si USA 1988; 85 : 5102-6.

76. Lichtenstein A, Berenson J, Gera JF, Waldburger K, Martinez-Maza O, Berek JS. Resistance of human ovarian cancer cells to tumor necrosis factor and lymphokine-activated killer cells : correlation with expression of $H E R 2 /$ neu oncogenes. Cancer Res 1990 ; 50 : 7364-70.

77. Lichtenstein A, Gera JF, Andrews J, Berenson J, Ware CF. Inhibitors of ADP. ribose polymerase decrease the resistance of HER2/neu-expressing cancer cells to the cytotoxic effects of tumor necrosis factor. $J$ Immunol 1991; 146: 2052-8.

78. Duerksen-Hughes P, Wold WSM, Gooding LR. Adenovirus E1A renders infected cells sensitive to cytolysis by tumor necrosis factor. J Immunol 1989 ; 143 : 4193-200.

79. Gooding LR, Sofola IO, Tollefson AE, Duerksen-Hughes P, Wold WSM. The adenovirus E3-14.7K protein is a general inhibitor of tumor necrosis factor-mediated cytolysis. J Immunol 1990 ; 145 : 3080-6.

80. Rotello RJ, Lieberman RC, Purchio AF, Gershenson LE. Coordinated regulation of apoptosis and cell proliferation by transforming growth factor beta 1 in cultured uterine epithelial cells. Proc Natl Acad Sci USA 1991; 88 : 3412-5.

81. Missero C, Filvaroff E, Dotto GP. Induction of transforming growth factor beta 1 resistance by the E1A oncogene requires binding to a specific set of cellular proteins. Proc Nall Acad Sci USA 1991; 88 : 3489-93.

\section{Sum mary}

Programmed cell death is a function of the cellular background

Recent results, going beyond the mere description and definition of programmed cell death or apoptosis, suggest the existence of a common program of cell death, whatever the death-inducing agent ; of multiple pathways leading to this program within the cell ; and of a major influence of the state of the cell on the outcome of potential death signalling. The existence of a control compartment is postulated within the cell. This control compartment can be modified, with consequences as to the interpretation of a subsequent death signal, upon differentiation ; it can also be modified by alternative signalling to the cell (e.g. through surface CD4 molecules) or by the expression of certain oncogenes. 